\title{
In situ investigation of the liquid/solid interface of a block copolymer solution under shear stress using microbeam grazing-incidence small-angle $x$-ray scattering
}

\author{
Andreas Timmann ${ }^{\text {a) }}$ and Stephan Volkher Roth \\ HASYLAB at DESY, Notkestrasse 85, 22607 Hamburg, Germany \\ Steffen Fischer and Stephan Förster \\ Institut für Physikalische Chemie, Universität Hamburg, Grindelallee 117, 20146 Hamburg, Germany
}

(Received 29 August 2007; accepted 31 October 2007; published online 19 November 2007)

\begin{abstract}
An aqueous solution of poly(isoprene ${ }_{55}$-block-ethylene oxide ${ }_{170}$ ) was investigated at different temperatures combining rheometry in plate/plate-geometry and microbeam grazing incidence small-angle x-ray scattering. In the vicinity of the rotator plate, we were able to separate the bulk scattering of the solution from the grazing incident signal stemming of the interface and to determine a thickness of an interface layer at the liquid-solid interface. We followed the thickness of the interface layer in situ as a function of shear rate. This kind of measurements can be important to decide if the first micellar layer sicks or slips at the surface under shear. (C) 2007 American Institute of Physics. [DOI: 10.1063/1.2815929]
\end{abstract}

Amphiphilic block copolymers are able to form various structures in a mixture with a selective solvent. ${ }^{1}$ These are, for example, micelles, wormlike micelles, and vesicles. At moderate and higher concentrations, the form face centered cubic (fcc) phases, hexagonal (hex) packed cylinders, or lamellas (lam). The structures have length scales from several nanometers up to several hundred nanometers. Applying external fields such as shear fields can dramatically change the overall structure of the sample. A well suited method that monitors these changes are small-angle neutron scattering (SANS) and small-angle x-ray scattering (SAXS). Except for some recent grazing incidence SANS measurements under shear, ${ }^{2,3}$ only transmission experiments were conducted on in situ combination of rheology and small-angle measurements. ${ }^{4-9}$ Because the interface of the colloidal solution inside a shear geometry is sometimes of special interest, we conducted an in situ combination of rheology with microbeam gracing incidence SAXS ( $\mu$-GISAXS). ${ }^{10}$

For our investigations, we used a $13 \mathrm{wt} \%$ solution of poly(isoprene ${ }_{55}$-block-ethylene oxide $\left.{ }_{170}\right)$ in water. The composition was determined by nuclear magnetic resonance and a narrow molecular weight distribution $M_{w} / M_{n}=1.004$ was determined by gel permeation chromatography based on poly(butadiene) calibrants. ${ }^{11}$ The block copolymer was synthesized by subsequent anionic polymerization of isoprene and ethylene oxide in tetrahydrofurane using a phosphazene base as initiator and water as termination agent. ${ }^{12}$ The solution was prepared by directly dissolving the polymer in water and stirring the mixture with a disperser, followed by one day storage at $45^{\circ} \mathrm{C}$. After that, the solution was kept in a refrigerator. The resulting solution was a slightly turbid, gelated liquid.

The scattering experiments were performed at the ultrasmall-angle scattering beamline BW4 at the Hamburger Synchrotronstrahlungslabor using the moderate microfocus. ${ }^{13}$ The monochromatic x-ray beam $(\lambda=0.1382 \mathrm{~nm})$

\footnotetext{
a) Author to whom correspondence should be addressed. Electronic mail:
} andreas.timmann@desy.de was collimated and focused using a beryllium compound refractive lens to a cross section $40 \times 20 \mu \mathrm{m}^{2}(W \times H)$ at sample position. The scattering patterns were recorded with a mar-CCD165 camera on a sample to detector distance of $2170 \mathrm{~mm}$.
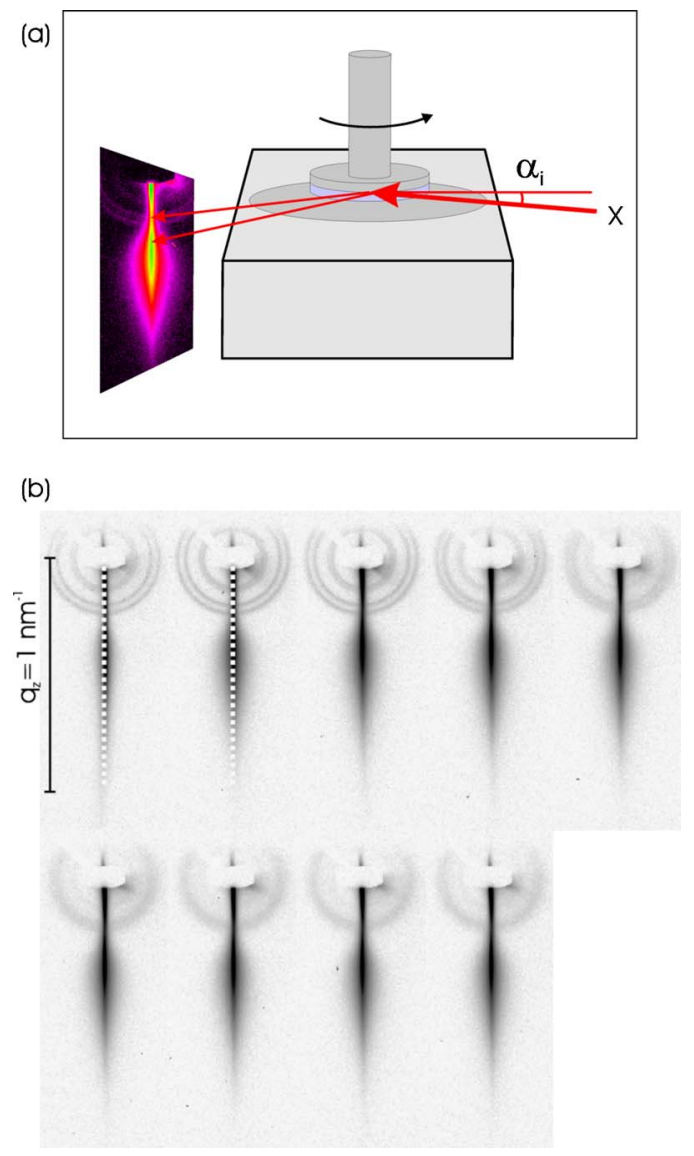

FIG. 1. (Color online) (a) Scheme of the experimental setup. X denotes the incoming x-ray beam and $\alpha_{i}$ the incidence angle. (b) Change of the scattering pattern at $T=15.4{ }^{\circ} \mathrm{C}$ with increasing shear rate $(\dot{\gamma}=0,5,12,55,164$, 208, 258, 360, and $475 \mathrm{~s}^{-1}$ ). The dotted lines indicate the detector cuts shown in Fig. 2. 
(a)

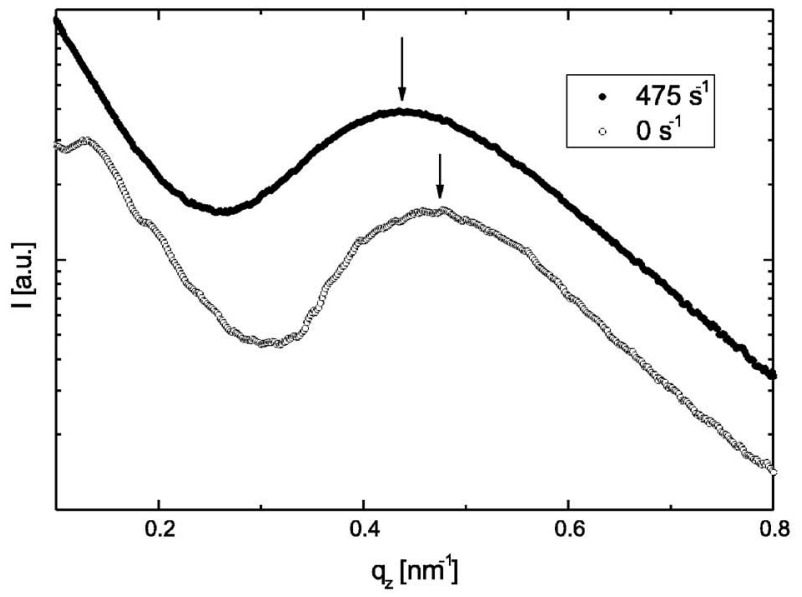

(b)

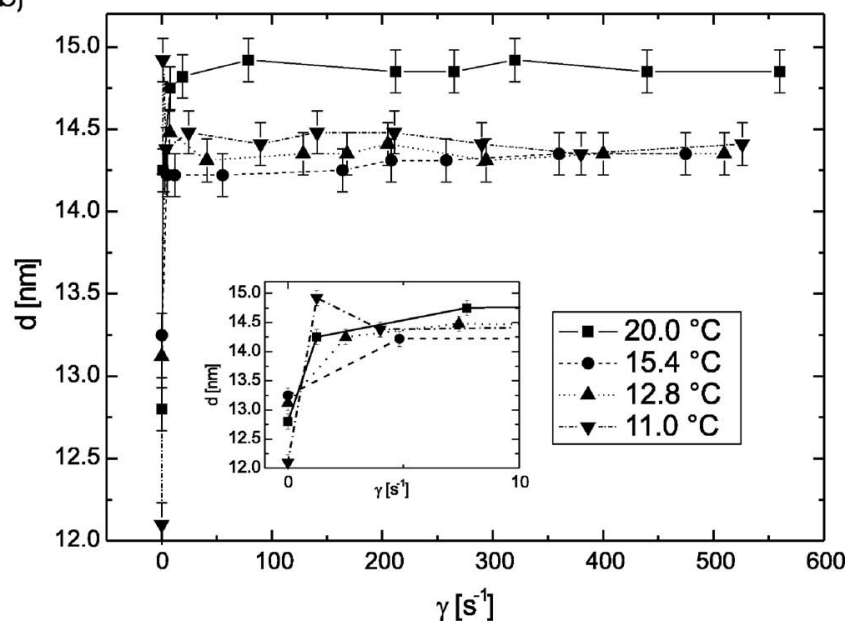

FIG. 2. (a) Detector cuts of the interface signal at $T=15.4{ }^{\circ} \mathrm{C}$. The arrows show the position of the maximum. (b) Calculated $d$ spacings vs shear rate at different temperatures (inset: initial response for $0 \mathrm{~s}^{-1}<\dot{\gamma}<10 \mathrm{~s}^{-1}$ ).

The sample was sheared with a standard plate-plate geometry of a Bohlin CVO rheometer equipped with a waterbased thermostat. The upper plate, on which the GISAXS measurements were taken, was made of polished stainless steel with a diameter of $20 \mathrm{~mm}$. Due to the high absorption of the water-based solution at the used wavelength, the measurements were taken at a distance of $0.4 \mathrm{~mm}$ ( $\gg$ beamsize) from the rim inside the sample. The direction of incident beam was perpendicular to the shear field. The incident angle was $\alpha_{i}=0.5^{\circ}$ [see Fig. 1(a)]. The measurements were conducted at temperatures of $20.0,15.4,12.8$, and $11.0^{\circ} \mathrm{C}$. The shear rates were about $\dot{\gamma}=0,5,12,55,165,200,300,400$, and $510 \mathrm{~s}^{-1}$. Deviations from these shear rates at the different temperatures are due to the equipment that was used in the experiments.

Figure 1(b) shows a series of measurements with an increasing shear rate at $15.4{ }^{\circ} \mathrm{C}$. In our measurements, we detect the scattering of the bulk as well as the scattering of the liquid-steel interface. In contrast to the interface scattering, the signal from the bulk is isotropic and centered around the primary beam position. Because the positions of both signals are well separated and the bulk scattering is weaker, it is easy to distinguish between them and analyze them independently. The change in the scattering pattern of the bulk signal is different from the interface signal.

Without a shear field, the bulk shows isotropic DebyeScherrer rings. This scattering pattern was identified as that of a fcc lattice. The broad inner ring originates from the (111) and (200) planes. The second ring is identified as the (220) and the third ring as (311) and (222) signals. From model calculations, we determine the lattice parameter to $a=78 \mathrm{~nm}$ and the core radius of the micelles to $R_{C}=11.7 \mathrm{~nm}$. Under an increasing shear rate the rings blur because of the decreasing size of the domains.

The interface signal on the other hand undergoes only one big change with the first application of shear. After that it stays nearly constant for every shear rate. To evaluate this change, detector cuts of the signal with and without shear were made. Because the scattered intensity of the interface is considerably larger than the intensity from the bulk, the detector cuts show practically only the scattering signal of the interface.

The detector cuts indicated in Fig. 1(b) by the dotted white lines are shown in Fig. 2(a). The small maxima at the beginning of the detector cut without shear are overlaying transmission signals. Therefore, they can be disregarded for the following consideration. The position of the maximum in the detector cut shifts from $q_{z}=0.477 \mathrm{~nm}^{-1}$ without shear to $q_{z}=0.436 \mathrm{~mm}^{-1}$ under maximum shear. This last position under shear is almost independent of the shear rate. In principle, this behavior was found at all four temperatures.

We interpret this maximum as a maximum of the height correlation function in the direction perpendicular to the plate. Hence, we assume as first approximation a Bragg-like behavior of the scattering from the interface, which leads us to calculate the layer thickness as

$$
d=\frac{2 \pi}{q_{z}}
$$

at the $q_{z}$ position of the maximum. These calculated $d$ spacings are shown in Fig. 2(b). The values without shear stress show a systematic change with temperature with a maximum of $13.25 \mathrm{~nm}$ at $T=15.4{ }^{\circ} \mathrm{C}$. The values under shear are noticeably larger than without shear. The $d$ spacings under shear are nearly constant at shear rates of $\dot{\gamma}>10 \mathrm{~s}^{-1}$. The inset in Fig. 2(b) shows the initial response of $d$ for low shear rates $\dot{\gamma}<10 \mathrm{~s}^{-1}$. Clearly, the response is nonlinear and strongly depends on the temperature. For $T=20.0{ }^{\circ} \mathrm{C}$ the average $d$ spacing is $14.85 \mathrm{~nm}$. At the other temperatures, the $d$ spacings are considerably smaller. For $T=15.4,12.8$, and $11.0^{\circ} \mathrm{C}$ the average $d$ spacings are $14.28,14.36$, and $14.43 \mathrm{~nm}$, respectively.

We interpret the growth of the interface layer as a shear induce detachment (see Fig. 3). Without shear, the corona blocks of the micelles are attached to the metal surface. When the shear starts, the normal forces pull the micelles a

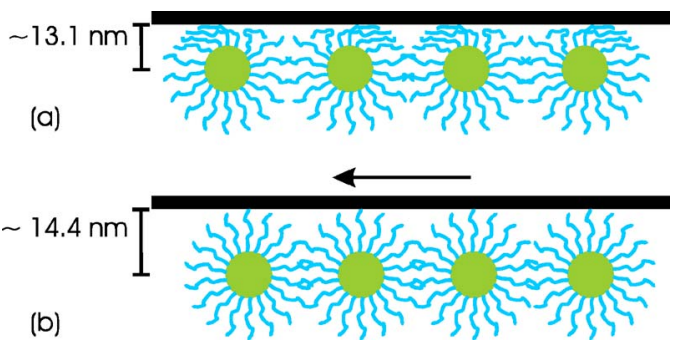

FIG. 3. (Color online) Exaggerated sketch of the detachment (a) without shear the corona blocks of the micelles get attached to the metal surface (b) with shear the corona blocks are detached from the metal surface. 
little bit away from the metal surface. This effect is reversible but retarded. The retardation times are in the order of tenth of minutes.

The distance of nearest neighbors in this fcc lattice is $d_{n}=a / \sqrt{2}=55.2 \mathrm{~nm}$. This leads to a thickness of the micellar shell of $R_{s}=d_{n} / 2-R_{c}=15.8 \mathrm{~nm}$. The thickness of the micellar shell at the metal surface without and under shear flow calculate to 1.4 and $2.7 \mathrm{~nm}$, respectively. This means there is a compressed adsorbate layer of polyethylene at the liquidsolid interface. This kind of layers have been described earlier by Wesemann et al. ${ }^{14}$ The dramatic growth of this layer under shear by a factor of 2 is an indicaton that the first micellar layer rather slips at the metal surface under shear then sticks to it.

We have shown that it is possible to measure the scattering signal of an interface between a bulk solution and a surface in reflection geometry. From the experiments mentioned above it is clearly shown that the behavior of bulk and steelliquid interface in a rheometric cell are quite different from each other. There is as strong indication, that the first micellar layer slips at the metal surface under shear. This thin interface layer will also be of special interest in applications concerning nanofluidics of many kinds. In future it would be interesting to measure with a higher shear rate resolution, to evaluate the deviation at low shear rates. It also will be interesting to do this kind of experiments with different rotator plate materials of different hydrophilicities or roughnesses such as polytetrafluoroethylene or silicon.

${ }^{1}$ Amphiphilic Block Copolymers, edited by P. Alexandridis and B. Lindman (Elsevier Science, Amsterdam, 2000), 1st edition, p. 1.

${ }^{2}$ M. Wolff, U. Scholz, R. Hock, A. Magerl, V. Leiner, and H. Zabel, Phys. Rev. Lett. 92, 255501 (2004).

${ }^{3}$ M. Wolff, A. Magerl, and H. Zabel, Physica B 357, 84 (2005).

${ }^{4}$ I. W. Hamley, Philos. Trans. R. Soc. London, Ser. A 359, 1017 (2001).

${ }^{5}$ P. Panine, M. Gradzielski, and T. Narayanan, Rev. Sci. Instrum. 74, 2451 (2003).

${ }^{6}$ J.-P. Habas, E. Pavie, C. Perreur, A. Lapp, and J. Peyrelasse, Phys. Rev. E 70, 061802 (2004).

${ }^{7}$ K. Mortensen, J. Polym. Sci., Part B: Polym. Phys. 42, 3095 (2004).

${ }^{8}$ S. Förster, M. Konrad, and P. Lindner, Phys. Rev. Lett. 94, 017803 (2005).

${ }^{9}$ E. Polushkin, S. Bondzic, J. de Wit, G. A. van Ekenstein, I. Dolbnya, W. Bras, O. Ikkala, and G. ten Brinke, Macromolecules 38, 1804 (2005).

${ }^{10}$ P. Müller-Buschbaum, Anal. Bioanal. Chem. 376, 3 (2003).

${ }^{11}$ A. Timmann, Ph.D. thesis, Universität Hamburg, 2005.

${ }^{12}$ S. Förster and E. Krämer, Macromolecules 32, 2783 (1999).

${ }^{13}$ S. V. Roth, R. Döhrmann, M. Dommach, M. Kuhlmann, I. Kröger, R. Gehrke, H. Walter, C. Schroer, B. Lengeler, and P. Müller-Buschbaum, Rev. Sci. Instrum. 77, 085106 (2006).

${ }^{14}$ A. Wesemann, H. Ahrens, R. Steitz, S. Förster, and C. A. Helm, Langmuir 19, 709 (2003). 